\title{
Hepatitis C Virus Perinatal Transmission
}

\author{
Umbeliana Barbosa de Oliveira \\ Emílio Ribas Institute of Infectious Diseases; São Paulo, SP, Brazil
}

The hepatitis C virus (HCV) is the most frequent cause of chronic hepatic disease. Its principal route of transmission is exposure to contaminated blood. Perinatal transmission is one of the less common modes of infection with HCV.

\section{Incidence}

Vertical transmission of HCV has been reported in numerous studies. However, the estimated rate of vertical transmission varies considerably due to several factors:

- Study methodology;

- Selection of maternal population;

- Risks involved in the transmission, such as coinfection with HIV and high levels of HCV RNA.

A review of the literature carried out in 1998 identified 976 children from 28 studies with follow-up periods that were sufficiently long to estimate the transmission rate. In those studies, the vertical transmission rate was below $10 \%$ in cases involving HIV-negative mothers. The risk of vertical transmission increased significantly in cases involving HIVpositive mothers. It is believed that, in these cases, the mother tends to present higher levels of HCV viremia, increasing the risk of transmission.

In a more recent review (published in 2001), the authors searched through studies published in the 1992-2000 period and identified 77 studies. They found clinical and demographic variables that influenced the transmission rate. They concluded that co-infection with HIV is the most significant factor associated with the risk of vertical HCV transmission. Findings regarding other possible risk factors, such as HCV genotype, type of delivery, and breastfeeding, have been inconclusive.

\section{Risk Factors}

As previously mentioned, the principal risk factor for vertical transmission of $\mathrm{HCV}$ is concomitant infection with HIV.

\section{Viremia Level}

Some studies have shown that viral load can be an important determinant factor for vertical transmission of HCV. It has been demonstrated that women with viral loads lower than $1 \times 10^{5}$ copies per $\mathrm{mL}$ have a lower risk of vertical transmission, whether co-infected with HIV or not. One study that compared viral loads among HCV RNA-positive mothers whose children were infected with HCV or not showed that, in cases of probable vertical transmission, the maternal viral load was ten times higher than in those cases in which transmission did not occur.

However, there are also inconsistent data in two large studies that found no significant differences between the HCV RNA levels of the mothers who transmitted HCV and those of mothers who did not.

The Brazilian Journal of Infectious Diseases $\quad$ 2007;11 (5) Suppl. 1:10-11. (C) 2007 by The Brazilian Journal of Infectious Diseases and Contexto Publishing. All rights reserved.

\section{Co-Infection with HIV}

Numerous studies have shown that the HCV vertical transmission rate is higher among HIV-positive women that among those who are HIV-negative (19\% vs. 4\%). This is in part explained by the fact that HIV-positive women present higher viral loads of HCV.

One study showed that the average level of HCV RNA in women co-infected with HIV was ten times higher than in women mono-infected with HCV. This shows that the increase in the co-infection risk can be nullified after adjusting the risk for the viral load, and antiretroviral therapy for HIV can reduce the risk of HCV transmission.

One study that compared HIV-positive women and HIVnegative women with similar viral loads of HCV showed that the risk of HCV transmission was similar in both groups. In that study, all HIV-positive women were receiving antiretroviral therapy.

Another study showed that children infected with HIV are at a higher risk of being infected with HCV than are those who are HIV-negative (17.1\% vs. 5.4\%). The explanation for these data remains unclear.

\section{Genotype}

There is no evidence that HCV genotype influences vertical transmission. The data collected to date do not allow us to establish a relationship between HCV genotype and vertical transmission risk.

\section{Breastfeeding}

It is possible to detect HCV RNA in breast milk and colostrum. However, HCV transmission through breastfeeding has not been documented. A likely explanation would be that $\mathrm{HCV}$ is inactivated by the effect of gastric acidity and that the HCV RNA levels in breast milk are very low.

Therefore, breastfeeding does not increase the risk that HCV will be transmitted from an infected mother to her child. The American College of Obstetricians and Gynecologists, as well as the American Academy of Pediatrics, allows breastfeeding by mothers who are infected with HCV.

It is important to emphasize that breastfeeding is not recommended in case of nipple fissure, due to the possibility of bleeding.

\section{Type of Delivery}

The impact that the type of delivery has on perinatal transmission of HCV is completely unknown. Vaginal delivery has been associated with an increase in the transmission risk. In a study carried out by Lin et al. in 1994, 70 pregnant women were evaluated. The vertical transmission rate among children who were born by vaginal delivery was $32 \%$, compared to $6 \%$ in cases of cesarean section. However, the high transmission rate in the Lin et al. study was associated with the fact that a large proportion of the women evaluated (76\%) were coinfected with HIV and HCV. Therefore, cesarean section is associated with a decrease in the HCV transmission risk in co- 
infected women. The higher risk of transmission in vaginal delivery might be associated with exposure of the newborn to HCV-contaminated blood during its passage through the vagina. However, the exposure to blood can sometimes be greater in cesarean section.

Many studies are needed in order to estimate the risk of perinatal transmission of HCV. Currently, cesarean sections are not recommended for pregnant women infected with HCV. Other risk factors associated with delivery are rupture of the placental membrane six hours or more before delivery and invasive procedures such as internal monitoring of the fetus (Blood 2000; 96:2045).

\section{Clinical Manifestations}

Newborns infected with HCV are generally asymptomatic. In these cases, a significant proportion presents normal alanine aminotransferase (ALT) levels.

One study monitored 104 children with vertical transmission upon birth for approximately 49 months (J Infect Dis 2000;181:419). Although HCV RNA levels were positive for $\mathrm{HCV}$ in $90 \%$ of these children, the clinical manifestations were rare. None of them presented hepatic insufficiency. The majority presented persistent or transitory increase in ALT levels. Levels of ALT were, in general, normal or slightly increased at birth, evolving to an increase in the fourth to sixth months of age, remaining high for two years, and declining significantly thereafter.

In that study, 20 children were submitted to hepatic biopsy. In all cases, evidence of chronic hepatitis was found. The degree of inflammation varied from mild to moderate. In three cases some level of fibrosis was found.

Other studies suggest that children infected with verticallytransmitted HCV are generally asymptomatic. However, such transmission is often accompanied by an increase in ALT levels during the first 6 to 12 months of life.

One large study monitored 266 perinatally HCV-infected children for an average of 4.2 years. In that study, approximately $20 \%$ of the children presented spontaneous clearance of HCV, and $80 \%$ evolved to chronic infection.

Clearance of HCV is defined as a loss in the polymerase chain reaction positivity for HCV RNA in those children who maintained detectable HCV RNA in the first year of life. Most of those children were asymptomatic.

Further studies involving long-term follow-up evaluation are needed in order to determine what proportion of children with chronic hepatitis $\mathrm{C}$ caused by vertical transmission will develop hepatic insufficiency and hepatocarcinoma.

\section{Diagnosis}

The initial diagnosis of infection with HCV in adults is made through anti-HCV antibody testing. However, this test presents limitations when used in children.

This is primarily due to the passage of IgG from the mother to the child through the placental barrier. Therefore, the presence of anti-HCV in the serum of the child does not necessarily indicate $\mathrm{HCV}$ infection. Clearance of the anti-HCV passively acquired from the mother can take more than 12 months, although it occurs within 12 months in 95\% of the cases.

Second, the presence of maternal anti-HCV does not necessarily mean that the mother is chronically infected with HCV.

A diagnosis of vertical transmission is made by identifying HCV RNA in the mother and child.

It is important to emphasize that the HCV RNA test can also present false-positive or false-negative results. A falsepositive result can occur as a result of contamination of positive samples. A false-negative result occurs due to a loss of RNA during sample storage or to an error in RNA extraction.

A consensus from the National Institutes of Health recommends that children born to mothers testing positive for HCV should be submitted to the HCV RNA test on two occasions: between 2 and 6 months of life and after 15 months of life (together with anti-HCV tests on the latter occasion).

\section{Conclusions}

The incidence of vertical transmission by $\mathrm{HCV}$ is approximately $2 \%$ to $5 \%$. The risk is higher in the following situations: co-infection with HIV; and high maternal viral load of HCV.

There are still no effective interventions to reduce the risk of HCV transmission from the mother to the child. As yet, $\mathrm{HCV}$ testing is not recommended for pregnant women.

\section{References}

1. Manzini P., Saracco G., Cerchier A., et al. Human immunodeficiency virus infection as risk factor for mother-tochild hepatitis $\mathrm{C}$ virus transmission; persistence of anti-hepatitis $\mathrm{C}$ virus in children is associated with the mother's anti-hepatitis C virus immunoblotting pattern. Hepatology 1995;21:328.

2. Thomas S.L., Newell M.L., Peckham C.S., et al. A review of hepatitis $\mathrm{C}$ virus (HCV) vertical transmission: risks of transmission to infants born to mothers with and without HCV viraemia or human immunodeficiency virus infection. Int $\mathrm{J}$ Epidemiol 1998;27:108.

3. Yeung L.T., King S.M., Roberts E.A. Mother-to-infant transmission of hepatitis C virus. Hepatology 2001;34:223.

4. Terrault N. Epidemiological evidence for perinatal transmission of hepatitis C virus. Viral Hepatitis Reviews 1998;4:245.

5. Lin H.H., Kao J.H., Hsu H.Y., et al. Possible role of high-titer maternal viremia in perinatal transmission of hepatitis $C$ virus. J Infect Dis 1994;169:638.

6. Kumar R.M., Shahul S. Role of breast-feeding in transmission of hepatitis $\mathrm{C}$ virus to infants of HCV-infected mothers. J Hepatol 1998;29:191.

7. Azzari C., Resti M., Moriondo M., et al. Vertical transmission of $\mathrm{HCV}$ is related to maternal peripheral blood mononuclear cell infection. Blood 2000;96:2045.

8. Tovo P.A., Pembrey L.J., Newell M.L. Persistence Rate and Progression of Vertically Acquired Hepatitis C Infection. J Infect Dis 2000; $181: 419$.

9. Vogt M., Lang T., Frosner G., et al. Prevalence and clinical outcome of hepatitis $\mathrm{C}$ infection in children who underwent cardiac surgery before the implementation of blood-donor screening. N Engl J Med 1999;341:866.

10. Three broad modalities in the natural history of vertically acquired hepatitis C virus infection. Clin Infect Dis 2005;41:45. 